Federal Reserve Bank of Minneapolis

Research Department Staff Report 304

April 2002

\title{
The Home Market and the Pattern of Trade: Round Three
}

\author{
Thomas J. Holmes* \\ University of Minnesota \\ and Federal Reserve Bank of Minneapolis \\ John J. Stevens* \\ Board of Governors \\ of the Federal Reserve System
}

\begin{abstract}
Does national market size matter for industrial structure? Round One (Krugman) answered in the affirmative: Home market effects matter. Round Two (Davis) refuted this, arguing that an assumption of convenience - transport costs only for the differentiated goods - conveniently obtained the result. In Round Three we relax another persistent assumption of convenience - two industry types differentiated only by the degree of scale economies - and find that market size reemerges as a relevant force in determining industrial structure.
\end{abstract}

*Holmes, University of Minnesota and Federal Reserve Bank of Minneapolis (holmes@econ.umn.edu). Stevens, Board of Governors of the Federal Reserve System (John.J.Stevens@frb.gov). Holmes acknowledges support from the NSF through grant SES 9906087. The views expressed herein are those of the authors and not necessarily those of the Federal Reserve Bank of Minneapolis, the Federal Reserve System, or the National Science Foundation. 


\section{Introduction}

A famous result due to Krugman (1980) is that increasing returns industries will tend to concentrate production within large markets. If a large country begins to trade with a small country, the large country will shift its industrial structure towards the production of increasing return type goods and export these to the small country. The small country, in turn, will shift its structure towards constant return type goods and export these to the large country. For example, if trade barriers are reduced between a small country such as New Zealand and a large country such as Japan, New Zealand will shift away from a scaleeconomy industry such as autos towards a constant returns sector such as wool. Krugman's result is important because the success of an economy is thought to be related, in part, to its industrial mix; New Zealand is unlikely to be rich completely specializing in wool.

In a recent $A E R$ paper, Davis (1998) reports a striking finding that overturns Krugman's result. In the original Krugman theory, transportation cost is assumed to be positive for the increasing returns sector and zero for the scale economy sector. The absence of transportation cost for the constant returns sector was regarded as an innocuous simplifying assumption. Davis shows first that this is an implausible assumption to make and, second, without this assumption Krugman's result is overturned. He shows that when transportation cost is the same for the two sectors, trade has no effect on a country's production mix between scale economy and constant returns sectors. To illustrate, consider the example of New Zealand and Japan and assume (somewhat implausibly) that there are no differences in Ricardian comparative advantage between the two countries or differences in factor proportions. The only difference is country size. Suppose the transportation cost of shipping wool is as high as the cost of shipping automobiles and automobile parts (which seems plausible). Then opening up trade will not lead New Zealand to shift production from autos to wool; there is no shift in the small country towards the constant returns sector.

This paper is a third round on the issue, and our results breathe new life into Krugman's original findings. We find that a seemingly innocuous simplifying aspect of the Davis model is actually crucial. Davis assumes there are two types of industries: one type is pure constant returns to scale and the other type has a fixed degree of scale economies. Rather 
than have two industry types, our model allows for a range of industry types that vary in the degree of scale economies. We follow Davis in assuming that the transportation cost is the same across industries. We find that industries with a low degree of scale economies are never traded, consistent with the Davis result that the constant returns sector is not traded. Goods with a medium and high degree of increasing returns are traded. Our key result is that the pattern of trade here does depend on country size, just like the original Krugman result. The small country exports the medium increasing-returns products, the large country exports the high increasing-returns products. Think about food processing as having moderate increasing-returns as opposed to wool (constant returns) and autos (high increasing returns). We find that opening of trade between New Zealand and Japan causes New Zealand to shift production into food processing out of autos, to export food processing and import autos, all the while leaving the wool sector alone.

To allow for variations in the degree of scale economies across industries, we are forced to step out of the standard Dixit-Stiglitz model of monopolistic competition, the workhorse model of this literature. This structure features a fixed cost and constant marginal cost. In the zero profit equilibrium, fixed costs as a share of revenues equals the markup share of price. This share depends upon the elasticity of demand but is independent of the technology parameters. Thus, increasing the fixed cost in a particular industry has no effect in increasing the equilibrium fixed cost share in that industry; the share remains constant. In fact, in the benchmark case where demand for final (composite) goods is Cobb-Douglas, a change in the fixed cost parameter of a particular industry has zero effect in all countries on the equilibrium allocation of inputs across industries. ${ }^{1}$ A particular concern is that as the fixed cost parameter goes to zero, there is no sense in which the outcome gets close to the constant returns case. It is plainly the case that varying the fixed cost parameter in the Dixit-Stiglitz world is not an interesting exercise.

We develop a structure to conduct our analysis that departs in two ways from the standard

\footnotetext{
${ }^{1}$ If the fixed cost doubles in an industry, in the new equilibrium there are half as many firms in that industry in each country as before and each firm produces twice as much output. Thus zero profit is maintained. The Cobb-Douglas assumption leaves industry revenue fixed, so the market clearing condition continues to hold.
} 
Dixit-Stiglitz world. First, the set of possible differentiated products is fixed and as a result there may be multiple entry, i.e., oligopoly, for any particular product. (With Dixit-Stiglitz there is at most one producer of each product.) Second, while the technology features an initial range of increasing returns, there exists a finite minimum efficient scale beyond which average cost is constant. (With Dixit-Stiglitz there is a fixed cost and constant marginal cost so the minimum efficient scale is infinity.) These two departures from the standard analysis complement each other; i.e., once we have made the first departure it helps considerably to make the second departure as well. The assumption that constant returns are reached at a large enough scale implies that in large markets the perfect competition outcome can be sustained. This allows for a structure that is quite tractable but rich enough to capture the importance of scale economies in small markets, given the initial range of increasing returns to scale.

In our model, products vary in the minimum efficient scale of production. Our question is how the structure of production and trade varies with a product's degree of scale economies. We begin our analysis by rederiving Krugman's and Davis' results in a special case of our alternative formulation. In this special case, industries can be one of two minimum efficient scale types. The first type all have minimum efficient scale equal to zero, i.e. constant returns. The second type all have a minimum efficient scale equal to an identical high level. For the Krugman case, we assume transportation cost is zero for the constant returns industry. We show that when a small country trades with a large country, the small country tends to specialize in the constant returns sector, while the large country specializes in the increasing returns goods. Thus we rederive Krugman's home market result, though there are some subtleties as we discuss in the main text. For the Davis case, we assume transportation cost is equal for the two sectors. We rederive Davis' result that there is no trade in the constant returns sector. All trade is in the increasing returns sector. Reductions in trade barriers have no effect on the structure of production.

The main body of our analysis considers the general case where there is a continuum of industry types ranging from zero minimum efficient scale to high minimum efficient scale. We find that the equilibrium with trade is characterized by two cutoffs, dividing industries into low, medium, and high returns to scale. Goods with low minimum efficient scale are 
not traded. Goods in the high minimum efficient scale range are not produced in the small country. The small country pays for imports of high range goods with exports of medium range goods. A reduction in the trade barrier reduces the range of goods produced in the small country.

This paper holds the transportation cost fixed and varies the degree of scale economies. Amiti (1998) uses the standard Dixit-Stiglitz structure to consider what happens when sectors vary in transportation cost but are identical in scale economies (and product differentiation). She shows that the small country tends to specialize in goods with low transport costs. $^{2}$ In a related paper (Holmes and Stevens, 2002), we use a variant of the model in this paper to further explore the effects of differences in transportation costs alone on the structure of production.

\section{The Model}

We begin by describing the environment and then define equilibrium.

\subsection{The Environment}

There is a continuum of industries indexed by $i$ on the unit interval, $i \in[0,1]$. Let $q(i)$ denote the consumption of good $i$. Consumers have Cobb-Douglas preferences,

$$
U=\int_{0}^{1} \ln (q(\tilde{\imath})) d \tilde{\imath}
$$

Labor is the only input in production. The production technology across industries varies by a parameter $\theta$. Given $\theta$, the labor requirement to produce $q$ units takes the following functional form:

$$
\ell=c(q, \theta)=\left\{\begin{array}{c}
\alpha \theta+(1-\alpha) \theta^{\frac{-\alpha}{1-\alpha}} q^{\frac{1}{1-\alpha}}, \quad \text { if } q<\theta \\
q, \quad \text { otherwise }
\end{array}\right.
$$

for a parameter $\alpha \in\left[\frac{1}{2}, 1\right) .^{3}$ The parameter $\theta$ is the minimum efficient scale of the industry.

\footnotetext{
${ }^{2}$ She also considers other comparisons, such as what happens when the small country and the large country differ in factor composition.

${ }^{3}$ The qualitative results hold for $\alpha \in\left(0, \frac{1}{2}\right)$ as well, but restricting attention to $\alpha \geq \frac{1}{2}$ simplifies by reducing the range of cases that need to be considered.
} 
For $q$ above $\theta$, average cost and marginal cost are constant at one labor unit. For $q$ below $\theta$, average cost is greater than one unit and falling while marginal cost is less than one and rising. For example, in the case where $\alpha=\frac{1}{2}$, average cost and marginal cost reduce to

$$
\begin{aligned}
A C(q, \theta) & =\frac{c(q, \theta)}{q}=\frac{1}{2}\left(\frac{q}{\theta}+\frac{\theta}{q}\right)>1, \quad q<\theta \\
M C(q, \theta) & =\frac{\partial c(q, \theta)}{\partial q}=\frac{q}{\theta}<1, \quad q<\theta .
\end{aligned}
$$

We motivate this formulation of the technology by an indivisibility. Suppose that in every industry labor must be used to complete two tasks. Let $\ell_{1}$ and $\ell_{2}$ denote the amount of labor allocated to each task and suppose total output is Cobb-Douglas,

$$
q=\lambda \ell_{1}^{\alpha} \ell_{2}^{1-\alpha}
$$

with $\lambda$ normalized to

$$
\lambda \equiv\left(\frac{\alpha}{1-\alpha}\right)^{1-\alpha}+\left(\frac{1-\alpha}{\alpha}\right)^{\alpha} .
$$

With this technology, the cost minimizing way to produce $q$ units of output requires $q$ units of labor, with a share $\alpha$ allocated to task 1 and a share $1-\alpha$ allocated to task 2 . Suppose there is an indivisibility constraint that

$$
\ell_{1} \geq \alpha \theta
$$

Then for $q>\theta$, the constraint is not binding and cost per unit is one labor unit. But for $q<\theta$, the constraint is binding. The firm has to employ $\alpha \theta$ units in task 1 even though it would prefer to use less. The first term of the cost function (2) is this fixed cost of the units employed at task 1. The second term is the variable cost of the units employed at task 2 .

While industries all have the same $\alpha$, they vary in $\theta$. Let $\theta(i)$ denote the minimum efficient scale in industry $i$. Let $f(\theta)$ denote the density of industries of type $\theta$ in the economy. Since the total measure of industries is one,

$$
\int_{0}^{\bar{\theta}} f(\theta) d \theta=1 .
$$

There are two countries, one small and one large. Let $L$ be total labor in the small country and $L^{*}$ be total labor in the large country. 
There is an iceberg transportation cost to ship goods from one country to the other. Assume that for industry $i, \tau(i) \geq 1$ units must be shipped for every one unit that arrives. The main focus will be the case where $\tau$ is constant across $i$ and strictly greater than 1 .

\subsection{Equilibrium}

Let $w$ be the wage in the small country and let $\Pi$ be the aggregate profit arising from all firms located in the small country. Then income in the small country is

$$
I=w L+\Pi .
$$

Analogously define income $I^{*}$ in the large country. Let $A \equiv\left(w, I, w^{*}, I^{*}\right)$ denote the set of aggregate variables.

The structure of behavior in this model is a combination of competition and oligopoly. All individuals are small relative to the economy as a whole so they take the aggregate variables in $A$ as fixed. Within any given industry, prices are set by oligopolist firms who take the aggregate variables $A$ as given. Since demand in each industry is unit elastic, prices set in other industries do not matter for the behavior of this industry, so the vector $A$ with wages and aggregate income is all that is needed.

There is a two stage game that is played in every industry $i$. In stage 1 , firms simultaneously make entry decisions. Let $n_{i}$ and $n_{i}^{*}$ be the number of firms in industry $i$ that commit to locating in the small and large countries. At the point of entry, firms commit themselves to satisfying the indivisibility constraint, $\ell_{1} \geq \alpha \theta(i)$. In stage 2 , firms simultaneously set prices, competing in a Bertrand fashion.

Our discussion of equilibrium has three parts. First we discuss the equilibrium of stage 2, the price-setting stage. Next we discuss the equilibrium of stage 1, the entry stage. Finally, we discuss the economy-wide equilibrium.

\subsubsection{The Price-Setting Stage}

In stage 2 , the entry decisions $\left(n, n^{*}\right)$ have already been determined (To simplify notation, the subscript for industry $i$ is implicit here). Firms observe entry as well as the aggregate variables $A \equiv\left(w, I, w^{*}, I^{*}\right)$. Firms can price discriminate and set a different price in each 
market. Let $p^{L}$ and $p^{E}$ be the local and export prices set by a firm in the small country. The delivered export price is $\tau p^{E}$. Let $q^{L}$ and $q^{E}$ denote a small country firm's output for the local and export markets and let $q=q^{L}+q^{E}$ be the total output. Analogously define $p^{L *}, p^{E *}, q^{L *}, q^{E *}$, and $q^{*}$ for a firm in the large country.

We restrict attention to symmetric equilibria where firms that locate in the same industry and the same country behave symmetrically. The price set by any given firm in stage 2 must maximize the firm's profits, taking as given the prices set by the other firms and the aggregate variables. In a separate appendix available on the web we show the following about equilibrium in the price subgame. ${ }^{4}$

Lemma 1 Given any $A$ and entry vector $\left(n, n^{*}\right)$, a symmetric equilibrium to the price subgame exists. If there are at least two firms in a given country, $n \geq 2$ or $n^{*} \geq 2$, there is a unique symmetric equilibrium of the price subgame. If each location has a single firm, $n=1$ and $n^{*}=1$, and if there is an equilibrium with trade, then it has a unique equilibrium.

When $n=1$ and $n^{*}=1$, there may exist a continuum of equilibria with no trade. For this case take some arbitrary equilibrium selection. For all other cases, the symmetric equilibrium is unique. Let $\pi\left(n, n^{*}\right)$ and $\pi^{*}\left(n, n^{*}\right)$ denote profits for a firm in the small and large country. Note these are implicitly functions of the industry $i$ and the aggregate vector A. It is immediate that

$$
\begin{aligned}
\pi(1,0) & =I+I^{*} \\
\pi\left(n, n^{*}\right) & \leq 0, n \geq 2 .
\end{aligned}
$$

To understand the first statement, observe that if a firm has a monopoly in the small country and it has no competitor in the large country, it can extract the entire industry revenue as profit by making the price arbitrarily large (recall demand is unit elastic). To understand the second statement, observe that if there are two firms at the same location, Bertrand competition drives price equal to marginal cost, which is no greater than average cost, so profits must be non-positive.

\footnotetext{
${ }^{4}$ The appendix can be accessed at http://www.econ.umn.edu/ ${ }^{\sim}$ holmes/research.html.
} 


\subsubsection{The Entry Stage}

Turn now to stage 1. In an equilibrium, all entrants receive nonnegative profit and there is no possibility of profit from further entry. Formally, equilibrium entry $\left(n, n^{*}\right)$ must satisfy

$$
\begin{aligned}
\pi\left(n, n^{*}\right) & \geq 0 \\
\pi\left(n+1, n^{*}\right) & \leq 0
\end{aligned}
$$

with analogous conditions on profits holding in the large country. Note these conditions do not require that there be zero profit in all industries. If an industry has positive profits as a monopoly but has negative profits as a duopoly, the industry will have positive profits in equilibrium.

In general there may be multiple equilibria in the entry game. To see this, consider a simple case where there is only a single country. If one firm enters it has a monopoly and it extracts the income $I$. If two firms enter, price is driven to marginal cost. Suppose the country is large enough so that the output of both firms exceeds the minimum efficient scale. Monopoly is an equilibrium here because if a second firm enters, it has zero profit, so there is no strict positive incentive to enter. But duopoly is also an equilibrium because both firms earn zero profit. For analogous reasons, there can be multiple equilibria to the entry game with two countries. In cases of multiple equilibria, we prefer to focus on equilibria where entry forces profits to zero. Hence we use the following elimination criterion.

Elimination Criterion 1. Suppose there is an equilibrium $\left(n, n^{*}\right)$ to the entry game such that $\pi\left(n, n^{*}\right)>0$. Suppose also that $\pi\left(n+1, n^{*}\right)=0$ and $\pi^{*}\left(n+1, n^{*}\right) \geq 0$, which implies that $\left(n+1, n^{*}\right)$ is an equilibrium. Eliminate $\left(n, n^{*}\right)$ from the set of equilibria to the subgame.

Even among the set of equilibria where all firms obtain zero profit, there may exist multiple equilibria which differ by price. In particular, if $I^{*}$ is large enough, if $I$ is small enough, and if $\tau w^{*} \leq w$, then we can construct an equilibrium with multiple firms in one location and none in the other, as well as a second equilibrium where this is reversed. These equilibria can be Pareto ranked. We eliminate the Pareto inferior equilibrium.

Elimination Criterion 2. Suppose there are two equilibria $\left(n, n^{*}\right)$ and $\left(\tilde{n}, \tilde{n}^{*}\right)$ to the entry game and that all profits are zero in both. If all consumers weakly prefer $\left(\tilde{n}, \tilde{n}^{*}\right)$, and some consumers strictly prefer it, then eliminate $\left(n, n^{*}\right)$ from the set of equilibria to the subgame. 
In our definition of equilibrium to the entry game, we restrict attention to equilibria that are not eliminated by criterion 1 or 2 .

\subsubsection{Economy-Wide Equilibrium}

We turn now to a definition of equilibrium for the economy as a whole. It consists of a list of aggregate variables $A=\left(w, I, w^{*}, I^{*}\right)$ and entry decisions $\left(n_{i}, n_{i}^{*}\right)$ for each industry $i$, and price and quantity outcomes $\left(p_{i}^{L}, p_{i}^{E}, p_{i}^{L *}, p_{i}^{E *}\right)$ and $\left(q_{i}^{L}, q_{i}^{E}, q_{i}^{L *}, q_{i}^{E *}\right)$ such that:

(1) Consumer demand maximizes utility.

(2) Given $A$, the entry and pricing decisions are a subgame perfect equilibrium of the oligopoly game that are not eliminated by criterion 1 or 2 .

(3) The income taken as given for the oligopoly games equals the total income in the economy, $I=w L+\Pi$ and $I^{*}=w L^{*}+\Pi^{*}$.

(4) Supply equals demand in the labor market.

\section{The Krugman and Davis Results}

This section rederives the results in the literature for our alternative model. Instead of having a continuum of different industries, we follow the earlier literature by having two industry types, type $a$ (agriculture) and type $m$ (manufacturing). Assume that $\theta_{a}=0$ and that $\theta_{m}>0$. Thus, agricultural industries have constant returns to scale while there is a range of increasing returns with manufacturing industries. Suppose that all industries $i \in\left[0, f_{a}\right]$ are type $a$ and all industries $i \in\left(f_{a}, 1\right]$ are type $m$. Therefore, a fraction $f_{a}$ of industries are type $a$ and a fraction $f_{m}=1-f_{a}$ are type $m$. Let $\tau_{a}$ and $\tau_{m}$ denote the iceberg transportation cost parameter for each industry.

\subsection{Round 1: Krugman}

In the Krugman case there is no transportation cost for agricultural goods so $\tau_{a}=1$. Manufacturers do have a transportation cost so $\tau_{m}>1$. We derive conditions under which a Krugman-like result is obtained in which the small country completely specializes in agriculture. 
Proposition 1 Suppose (i) $L<\frac{\theta_{m}}{2}$, (ii) $L^{*}>2 \theta_{m}$, and (iii) $f_{m} L<f_{a} L^{*}$. Then there exists an equilibrium such that

- $w=w^{*}=1$.

- $n^{*}(i)=2$, for all $i$. (There is multiple entry in the large country for all goods.)

- $n(i)=2$, for $i \in\left[0, f_{a}\right], n(i)=0$, for $i \in\left(f_{a}, 1\right]$. (In the small country, there is multiple entry for each agricultural good, but zero entry for each manufactured good.)

- $p_{a}^{*}=p_{m}^{*}=1$.

- $p_{a}=1, p_{m}=\tau_{m}$.

Conditions (i) and (ii) ensure that the small country is sufficiently small and that the large country is sufficiently large. Condition (iii) ensures that agriculture is a large enough share of the budget to make it possible for the small country to completely specialize in agriculture. (Note that if we were to assume $f_{a} \geq \frac{1}{2}$, then (i) and (ii) would imply (iii).)

We sketch the proof. The agricultural sector is constant returns. Given duopoly entry for all agricultural products in both countries, Bertrand competition ensures price equals marginal cost. Given duopoly entry for all manufacturing products in the large country, price equals marginal cost there as well. The assumption $L^{*}>2 \theta_{m}$ guarantees that the two firms will produce above minimum efficient scale, so marginal cost will equal 1 and that will equal the price. The large country is in a constant returns world and its prices are unaffected by trade with the small country. Consumers face a price of 1 for both kinds of goods. Consumers in the small country pay 1 for the agricultural goods but have to pay $\tau_{m}$ to import manufactured goods.

The only thing that remains to be checked is the profitability of entry by a manufacturing firm in the small country. If a firm enters, its local sales will equal

$$
\begin{aligned}
q^{L} & =\frac{I}{p^{L}} \\
& =\frac{L}{\tau_{m}} .
\end{aligned}
$$


The first equality follows from the Cobb-Douglas assumption; the second substitutes in $I=L$ (wage is 1 and there are no profits) and the fact that $p^{L}=\tau_{m}$ (since the firm will limit price, matching the delivered import price). In the export market, the entrant would set an export price of $p^{E}=\frac{1}{\tau_{m}}$, so that with transport costs it would match the price offered by the local firms in the large country. At this price, the entrant could choose to export any quantity $q^{E}$ that did not exceed $\tau L^{*}$. ( $L^{*}$ is the local demand in the large country at the local price of one; if the small-country firm exports $\tau L^{*}$ units then $L^{*}$ units are delivered.) The problem of an entrant is

$$
\begin{aligned}
\pi & =\max _{q^{E} \in\left[0, \tau L^{*}\right]} p^{L} q^{L}+p^{E} q^{E}-c\left(q^{L}+q^{E}\right) \\
& =\max _{q^{E} \in\left[0, \tau L^{*}\right]} L+\frac{1}{\tau_{m}} q^{E}-c\left(\frac{L}{\tau_{m}}+q^{E}\right) .
\end{aligned}
$$

Note that even though the export price is less than the minimum average cost of 1 , the entrant still might want to export because its marginal cost will be less than 1. But condition (i), $L<\frac{\theta_{m}}{2}$, is a sufficient condition for the maximized profit to be strictly negative, so there is no incentive to enter. It is clear that profit will be negative if $L$ is small. When the local market is close to zero, virtually all the entrant's revenues will come from exports. But the export price is below minimum average cost, so the entrant cannot break even with exports alone.

Proposition 1 confirms the Krugman intuition for this model when a small country is paired up with a large county. A subtlety arises when a medium size country is paired with a large country. Here a reversal is possible. We illustrate this with an example. The smaller of the two countries will be called the "medium" country because the size is intermediate between $\frac{\theta_{m}}{2}$ and $\theta_{m}$.

Example. Suppose the parameters are such that

1. $\frac{\theta_{m}}{2}<L<\theta_{m}$

2. $3 \theta_{m}<L^{*}$,

3. $\tau_{m}^{2}<\left[2-\frac{\theta_{m}}{L}\right]^{-1}$,

4. $\left[\frac{\theta_{m}}{L}-1+\frac{1}{\tau_{m}^{2}}\right]^{-1}<f_{m}$. 
Then there exists an equilibrium where the medium size country completely specializes in manufacturing, and the wage in the medium country exceeds the wage in the large country, $w>w^{*}$. A subset of the manufactured products are produced in the medium country by a monopolist (i.e. $n(i)=1$ for $i$ in some subset of $\left.\left(f_{a}, 1\right]\right)$. The remaining manufactured products are not produced in the medium country, nor are agricultural products produced, $n(i)=0, i \in\left[0, f_{a}\right]$. The large country has duopoly producers for all goods.

In this example, there are monopoly producers of manufactured goods in the medium country, but they get zero profit (the wage goes above 1 to drive this profit to zero). The medium country exports some manufactured goods and imports the other manufactured goods, as well as all the agricultural goods. It is easy to understand why this happens. As we increase $L$, the size of the smaller country, eventually there will be production of some manufactured goods in the smaller country. But for a medium size country, the local demand might be on the short side. By exporting, the medium size country can get to efficient scale. This does not adversely affect producers in the large country because they are already well above efficient scale.

\subsection{Round 2: Davis}

Davis considers the case where agricultural and manufactured goods have the same transportation cost, $\tau_{a}=\tau_{m}=\tau>1$. He shows in his model that there can never be equilibrium trade in agricultural goods. The intuition of his result is quite simple. Suppose the small country were to export agricultural goods to the large country. The small country must then have the lowest cost to produce the agricultural good, i.e., $\tau w \leq w^{*}$. Since the large country imports agricultural goods, and because there can be no two-way trade in agriculture, it must be that the large country is exporting manufactured goods. But now suppose a manufacturer from the large country shifts production to the small country. The costs of serving the consumers in the large country will not increase, since the wage advantage more than compensates for the additional transportation costs. But the costs of serving consumers in the small country go down because of the wage advantage and the fact that transport costs are now avoided. So profit strictly increases, contradicting the optimality of the manufacturer locating in the large country. 
Extending his result to our model involves some complications because our technology is different and because we have an oligopoly for any particular differentiated product as opposed to monopoly. Nonetheless, the same basic argument applies here. We state our formal proposition here and relegate the proof to the separate appendix.

Proposition 2 With equal transportation costs, $\tau_{a}=\tau_{m}=\tau>1$, there is no equilibrium under the Pareto dominance selection criterion with trade in agricultural goods. In both countries, the labor share in manufacturing equals $f_{m}$, so the industrial structure is the same.

\section{Round 3: The Continuum Model}

We now return to our general model with a continuum of industry types $\theta$. From the discussion of the Krugman model, we know that complications arise when the two countries are relatively close in size (recall the discussion of the case where a medium sized country trades with a large country).

To sharpen the analysis we focus on the case where the smaller country is quite small, and the larger country is quite large. The upper bound on the small country size is

$$
L<\int_{0}^{\bar{\theta}} \alpha \theta f(\theta) d \theta
$$

Under this bound, the total labor in the small country is insufficient to pay the fixed cost of opening positive production in all of the industries. The lower bound on the large country size is

$$
L^{*}>3 \bar{\theta}
$$

This assumption implies that the large country is big enough without trade to support three firms above minimum efficient scale in all the industries. By making the market big enough for three firms, we ensure that if there is a firm in the small country producing the good, there is still sufficient demand in the large country to support two firms (which we want to sustain the Bertrand outcome in the large country). 
Define a Large Country Perfect Competition Equilibrium to be one where $n_{i}^{*}=2$ for all industries $i$; i.e., there are two firms represented in every industry in the large country. In this section we restrict attention to equilibria in this class.

Proposition 3 There exists a Large Country Perfect Competition Equilibrium. This equilibrium is characterized by autarchy in goods with low scale economies; small country exports in goods with intermediate scale economies; and large country exports in goods with high scale economies.

The analysis underlying our proposition has two parts. First, we characterize how the market structure (number of firms) varies with $\theta$. Second, we show how the trade structure varies with $\theta$. Below we sketch our argument and put the formal proof in the appendix.

\subsection{Market Structure}

The market structure in the small country is characterized by two cutoffs, $\hat{\theta}_{1}<\hat{\theta}_{0}$. For

industries with $\theta$ below $\hat{\theta}_{1}$ there are two firms setting price equal to marginal cost a la Bertrand. Industries between $\hat{\theta}_{1}$ and $\hat{\theta}_{0}$ have a single firm. Industries above $\hat{\theta}_{0}$ have zero firms. The cutoffs are illustrated in Figure 1.

Our formal characterization of the equilibrium market structure proceeds in four steps. These steps characterize the market structure and show the existence of an equilibrium.

Step one is the observation that, in any equilibrium, the small-country wage $w$ satisfies the following bounds that depend upon the large-country wage and the transportation cost parameters,

$$
\frac{1}{\tau} w^{*}<w<\tau w^{*}
$$

The intuition for these bounds follows the discussion of the Davis result in Section 3.2.

Step two takes the aggregate state $A=\left(w, I, w^{*}, I^{*}\right)$ as given and determines the equilibrium to the oligopoly game for each type of industry $\theta$. We have set $w^{*}=1$ as the numeraire. In a Large Country Perfect Competition Equilibrium there are zero profits in the large country so total income there is wage income, $I^{*}=L^{*}$. Since the small country income equals $I=w L+\Pi$, we can represent the aggregate state by $(w, \Pi)$, the small country wage and the small country aggregate profit. 
Let $\pi(\theta, w, \Pi)$ be the profit of a monopolist in the small country given the industry scale parameter $\theta$ and the aggregate state $(w, \Pi)$. Analogous to the earlier formula (5), it equals local revenues plus export revenues minus total cost at the optimum export level,

$$
\pi(\theta, w, \Pi)=\max _{q^{E} \in\left[0, \tau L^{*}\right]}(w L+\Pi)+\frac{1}{\tau} q^{E}-w c\left(\frac{w L+\Pi}{\tau}+q^{E}, \theta\right) .
$$

It is immediate that $\pi$ is nonincreasing in the scale parameter $\theta$. Define $\hat{\theta}_{0}(w, \Pi)$ to be the maximum level of $\theta$ such that $\pi(w, \Pi) \geq 0$. Profit is negative for $\theta$ above $\hat{\theta}_{0}(w, \Pi)$ so there is zero entry above this threshold. Define

$$
\hat{\theta}_{1}(w, \Pi)=\frac{1}{2}\left(\frac{w L+\Pi}{w}\right) .
$$

At this point, when there are two entrants, the equilibrium output of each firm with Bertrand competition exactly equals minimum efficient scale. For $\theta$ less than $\hat{\theta}_{1}$, two firms enter in equilibrium. For $\theta$ between $\hat{\theta}_{1}$ and $\hat{\theta}_{0}$ a single firm enters.

The third step solves for the value of aggregate profits $\Pi$ as a function of the wage rate, taking into account that entry depends upon $\Pi$. Specifically, $\Pi(w)$ solves $H(\Pi, w)=0$ where

$$
H(\Pi, w) \equiv \Pi-\int_{\hat{\theta}_{1}(w, \Pi)}^{\hat{\theta}_{0}(w, \Pi)} f(\theta) \pi(\theta, w, \Pi) d \theta .
$$

We show in the separate appendix that for any $w$ in the relevant range (8), there is a unique solution.

The fourth step is to solve for the wage rate in the small country that clears the labor market. Define the excess demand for labor to be

$$
E(w)=\int_{0}^{\hat{\theta}_{0}(w, \Pi(w))} f(\theta) c(q(\theta, w), \theta) d \theta-L
$$

where $q(\theta, w)$ is the quantity of type $\theta$ good produced given wage $w$ and profit $\Pi(w)$. In the appendix we show that $E(w)$ is strictly positive near the lower bound of $w=\frac{1}{\tau}$ and strictly negative near the upper bound of $w=\tau$; continuity ensures that an equilibrium wage exists. Finally, note that in equilibrium $\hat{\theta}_{0}<\bar{\theta}$ since otherwise assumption (6) would imply the resource constraint is violated. 


\subsection{Trade Structure}

The equilibrium trade structure is characterized by two cutoffs, $\hat{\theta}_{E}$ and $\hat{\theta}_{0}$. The cutoff $\hat{\theta}_{0}$ is the same cutoff from above that separates the single-firm entry and zero entry cases. The cutoff $\hat{\theta}_{E}$ satisfies $\hat{\theta}_{E} \geq \hat{\theta}_{1}$ and $\hat{\theta}_{E}<\hat{\theta}_{0}$. For industries $\theta<\hat{\theta}_{E}$ there is no trade. For industries in the range $\theta \in\left(\hat{\theta}_{E}, \hat{\theta}_{0}\right)$ the small country producers all export. For industries $\theta>\hat{\theta}_{0}$, there is no production in the small country and all the small country demand is met by imports. These cutoffs are illustrated in Figure 1.

Recall that for $\theta<\hat{\theta}_{1}$, there are two producers in the small country and both firms produce in the constant-returns-to-scale region of the production function. There is no trade in these goods following the original Davis argument. The price of such goods in the small country equals $w$, minimum average cost. Since the equilibrium wage $w$ lies between $\frac{w^{*}}{\tau}$ and $\tau w^{*}$, there is no scope for trade.

For $\theta$ between $\hat{\theta}_{0}$ and $\bar{\theta}$, there are no producers in the small country. So demand is met by imports.

Now consider the range between $\hat{\theta}_{1}$ and $\hat{\theta}_{0}$ where there is a single producer in the small country. Examining the firm's problem (9), the firm will choose to export if the export price exceeds marginal cost at zero exports; i.e., if

$$
\frac{1}{\tau}>w \frac{\partial c\left(q_{L}, \theta\right)}{\partial q}
$$

for $q_{L}=\frac{w L+\Pi}{\tau}$. For $\theta<q_{L}$, marginal cost is constant in $\theta$, while it decreases in $\theta$ for $q_{L}<\theta$. (See equation (3).) Thus if any $\theta^{\circ}$ chooses to export, all $\theta>\theta^{\circ}$ also strictly prefer to export. Thus the export decision will have the form of a cutoff where all $\theta$ above the cutoff choose positive exports. Since there is a positive measure of imports there must be a positive measure of exports. Therefore, there must exist a cutoff $\hat{\theta}_{E}<\hat{\theta}_{0}$ where all $\theta$ above $\hat{\theta}_{E}$ export.

\section{Concluding Remarks}

Our analysis assumes the structure of demand is the same across products and varies the degree of scale economies across products. It is straightforward to see that analogous results 
would be obtained if the cost structure were the same across products but demand structure were to differ. In particular suppose that consumers have relatively greater demand for some products relative to others in the sense that they would choose to buy a larger quantity at the same price. For goods with a high enough level of demand, it might be possible even in the small country for firms to get to the constant returns to scale region, so these goods would not be traded, analogous to the way there is autarchy in our original model for the goods with low minimum efficient scale. Following the same logic as our original model, the small country will tend to export goods of intermediate level of demand and import goods with low levels of demand. One can think of the products with low level of demand as "boutique" or "niche" items. Only the large market can sustain production of these "unusual" items.

For the sake of tractability, we chose a production technology that features constant returns above some minimum efficient scale and we focus on the case where the large country is sufficiently large to have perfect competition throughout all the industries. Alternatively, we could have conducted an analysis with a constant marginal cost and a fixed cost that varied across industries. ${ }^{6}$ With this alternative structure, there would be at most one producer of a given product at each location, because of the usual Bertrand logic. It is intuitive that for low fixed cost products, there would be entry at both locations, so such goods would not be traded, analogous to our results here. For higher fixed cost goods, production would be only at one location. As is typical in oligopoly games, there are multiple equilibria in this environment regarding the country in which a given industry locates. Thus, there is no hope that a clean partition of industry and trade structure as in our proposition would be the equilibrium here. Nonetheless, we expect that a qualitative aspect of our result would continue to hold. In particular, we conjecture that if the highest fixed cost is high enough and if the large country is large enough, the highest fixed cost goods would only be produced in the large country. Thus the structure of production and trade would depend in systematic ways upon the size of the home market.

\footnotetext{
${ }^{5}$ See Holmes (1999) for a related analysis.

${ }^{6}$ If we had gone this way, we would also have assumed an elasticity of substitution between products greater than one rather than one, to bound the optimal monopoly price.
} 


\section{References}

Amiti, Mary, "Inter-Industry Trade in Manufactures: Does Country Size Matter?" Journal of International Economics, 44 (1998) 231-255.

Davis, Donald R., "The Home Market, Trade, and Industrial Structure," American Economic Review, 88 (1998), 1264-1276.

Holmes, Thomas J., "Scale of Local Production and City Size," American Economic Review Papers and Proceedings, 89 (1999), 317-20.

Holmes, Thomas J. and John J. Stevens, "Geographic Concentration and Establishment Size: Analysis in an Alternative Economic Geography Model," unpublished manuscript (2002).

Krugman, Paul, "Increasing Returns and Economic Geography," Journal of Political Economy, 99 (1991), 483-499.

Krugman, Paul, "Scale Economies, Product Differentiation, and the Pattern of Trade," American Economic Review, 70 (1980), 950-959. 
Figure 1: Small Country Market and Trade Structures in the Continuum Model

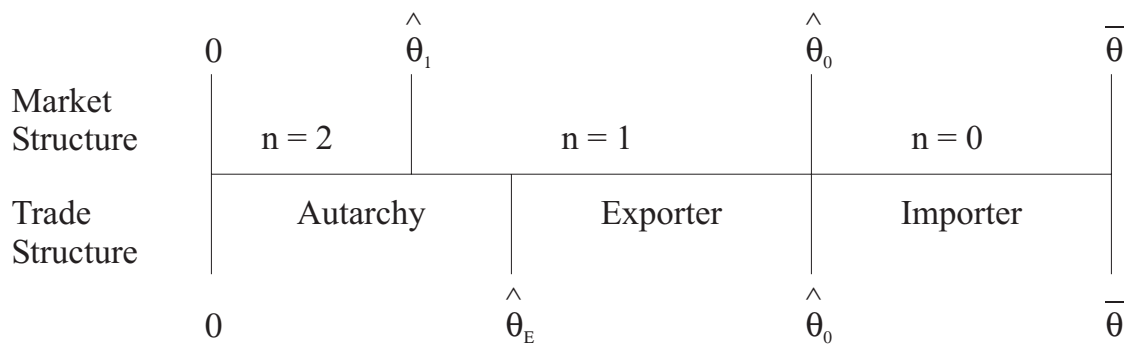

